\title{
Optically Tunable Resistive-Switching Memory in Multiferroic Heterostructures
}

\author{
Ming Zheng, ${ }^{1,2,}{ }^{*}$ Hao Ni, ${ }^{2,3}$ Xiaoke Xu, ${ }^{4}$ Yaping $\mathrm{Qi},{ }^{2}$ Xiaomin $\mathrm{Li},{ }^{4}$ and $\mathrm{Ju} \mathrm{Gao}^{2,5}$ \\ ${ }^{1}$ Department of Applied Physics, The Hong Kong Polytechnic University, Kowloon, Hong Kong \\ ${ }^{2}$ Department of Physics, The University of Hong Kong, Pokfulam Road, Hong Kong \\ ${ }^{3}$ College of Science, China University of Petroleum, Qingdao 255680, China \\ ${ }^{4}$ State Key Laboratory of High Performance Ceramics and Superfine Microstructure, \\ Shanghai Institute of Ceramics, Chinese Academy of Sciences, Shanghai 200050, China \\ ${ }^{5}$ School of Optoelectronic Engineering, Zaozhuang University, Zaozhuang, Shandong 277160, China
}

(Received 26 November 2017; revised manuscript received 4 March 2018; published 26 April 2018)

Electronic phase separation has been used to realize exotic functionalities in complex oxides with external stimuli, such as magnetic field, electric field, current, light, strain, etc. Using the $\mathrm{Nd}_{0.7} \mathrm{Sr}_{0.3} \mathrm{MnO}_{3} / 0.7 \mathrm{~Pb}\left(\mathrm{Mg}_{1 / 3} \mathrm{Nb}_{2 / 3}\right) \mathrm{O}_{3}-0.3 \mathrm{PbTiO}_{3}$ multiferroic heterostructure as a model system, we investigate the electric field and light cocontrol of phase separation in resistive switching. The electricfield-induced nonvolatile electroresistance response is achieved at room temperature using reversible ferroelastic domain switching, which can be robustly modified on illumination of light. Moreover, the electrically controlled ferroelastic strain can effectively enhance the visible-light-induced photoresistance effect. These findings demonstrate that the electric-field- and light-induced effects strongly correlate with each other and are essentially driven by electronic phase separation. Our work opens a gate to design electrically tunable multifunctional storage devices based on multiferroic heterostructures by adding light as an extra control parameter.

DOI: 10.1103/PhysRevApplied.9.044039

\section{INTRODUCTION}

The past decades have witnessed striking discoveries in strongly correlated complex oxides, typified by the emergence of high-temperature superconductivity [1], colossal magnetoresistance [2], electroresistance [3], metal-insulator transition [4], etc., which is deeply associated with microscopic electronic phase separation (EPS). By controlling the strength of the EPS, i.e., the relative proportion of coexisting multiphases, in these complex oxides using various external stimuli, such as electric field, current, magnetic field, strain, light, etc., exotic functionalities are highly desired to develop energy-efficient multifunctional devices. As one of the most fascinating correlated oxides, perovskite manganites display large-scale EPS in the charge-ordering ( $\mathrm{CO}$ ) antiferromagnetic insulating phase, the ferromagnetic (FM) metallic phase, and/or the paramagnetic (PM) insulating phase [5-10]. Because of the strongly coupled nature of the lattice, charge, spin, and orbital degrees of freedom, the external stimuli can tip the subtle balance of free energy between the coexisting phases in manganites, leading to dramatic changes in physical properties. For example, Thiele et al. [11] and Zheng et al. [12] in situ imposed an in-plane strain to $\mathrm{La}_{0.7} A_{0.3} \mathrm{MnO}_{3}$ $(A=\mathrm{Ca}, \quad \mathrm{Sr}, \quad \mathrm{Ba})$ films grown on ferroelectric

*zhengm@mail.ustc.edu.cn
$\mathrm{Pb}\left(\mathrm{Mg}_{1 / 3} \mathrm{Nb}_{2 / 3}\right) \mathrm{O}_{3}-\mathrm{PbTiO}_{3}$ (PMN-PT) single crystals via the linear piezoelectric response and obtained strain-mediated electric-field control of phase separation, magnetic, and transport properties. However, the lattice strain and physical properties decay to the initial states upon removing the controlled electric field. From a device application point of view, the voltage-pulse-induced nonvolatile tuning of physical parameters is indispensable. Fortunately, it has been realized in $R_{1-x} A_{x} \mathrm{MnO}_{3}(R=\mathrm{La}, \mathrm{Pr} ; A=\mathrm{Ca}, \mathrm{Sr}$, $\mathrm{Ba} / \mathrm{PMN}-\mathrm{PT}$ systems [13-15] in a stable and reversible manner using nonlinear lattice strain effects originating from non- $180^{\circ}$ ferroelastic domain switching. In addition, light illumination, conveniently, can induce the delocalization of $e_{g}$ carriers in the CO or PM insulating phase and demagnetization effect in the FM metallic phase, which significantly affects the magnetic interaction and phase balance of manganites. For example, Guo et al. [16] and Li et al. [17] reported opposite effects of light on resistance above and below Curie temperature in $\mathrm{La}_{0.8} \mathrm{Ca}_{0.2} \mathrm{MnO}_{3}$ and $\mathrm{Pr}_{0.65}\left(\mathrm{Ca}_{0.75} \mathrm{Sr}_{0.25}\right)_{0.35} \mathrm{MnO}_{3}$ films. Despite the efforts devoted to in situ manipulation of physical properties of manganite films using either electric field or light, the combined electric-field and light control (rather than the far more energy-consuming use of charge currents and/or magnetic fields) of EPS and the resultant functionalities are still very limited. There is no doubt that a systematic investigation of multifield tuning of EPS and physical 
properties would help to elucidate the optoelectronics physics and coupling effect of the electric field and the light in perovskite manganites, which is also important for designing energy-efficient multifunctional memory devices by exploiting additional degrees of freedom (i.e., photons).

$\mathrm{Nd}_{0.7} \mathrm{Sr}_{0.3} \mathrm{MnO}_{3}$ (NSMO) is one of the typical colossal magnetoresistive materials whose EPS is extremely sensitive to its strain state. A phase transition from the FM metallic to the $\mathrm{CO}$ insulating state can be observed by substrate-induced in-plane tensile strain with a decreasing thickness from 60 to $10 \mathrm{~nm}$ in NSMO films grown on $\mathrm{SrTiO}_{3}$ single crystals [18]. In this work, phase-separated NSMO thin films are epitaxially grown on ferroelectric PMN-PT(111) single-crystal substrates. We in situ modified the phase separation and resistance state of the NSMO films not only by the electric-field-induced ferroelastic strain effect but also by the light-induced delocalization effect, which results in a multilevel resistive-switching memory and adjustable photoresponse. Such a multiferroic heterostructure inherits the properties of voltage-pulse tunable nonvolatile storage devices and is also suitable for light-sensing applications.

\section{EXPERIMENTAL DETAILS}

The NSMO thin films are fabricated onto (111)-oriented PMN-PT single-crystal substrates using pulsed laser deposition from a high-purity stoichiometric ceramic target. The target is ablated using a $\mathrm{XeCl}(\lambda=308 \mathrm{~nm})$ excimer laser with a flux of $3 \mathrm{~J} / \mathrm{cm}^{2}$ and a repetition rate of $4 \mathrm{~Hz}$. The growth is conducted under a process pressure of 200 mtorr of pure $\mathrm{O}_{2}$ at a substrate temperature of $700{ }^{\circ} \mathrm{C}$, followed by in situ annealing in pure $\mathrm{O}_{2}$ under 750 torr for $1 \mathrm{~h}$ to remove oxygen vacancies and improve crystallinity. The film thickness $(36 \mathrm{~nm})$ is controlled by the number of laser pulses.

The crystal structure, phase purity, and epitaxial properties of the films are analyzed by a high-resolution Bruker D8 Discover X-ray diffractometer (XRD) equipped with $\mathrm{Cu}$ $K_{\alpha 1}$ radiation $(\lambda=1.5406 \AA)$. A Keithley 6487 voltage source is employed to apply an electric field across the PMN-PT substrate through the conducting NSMO film and the bottom silver electrode. A semiconductor laser with a wavelength of $532 \mathrm{~nm}$ and a power density of $5 \mathrm{~mW} / \mathrm{cm}^{2}$ serves as the light-illumination source. The resistance of the films is measured using the standard four-probe method in a closed-cycle cryostat. Figures 1(a)-1(c) illustrate the schematic of the experimental setups for in situ measurements of resistance under electric field and light illumination (using the standard four-probe method), electric-fieldinduced out-of-plane strain (using in situ XRD $\theta-2 \theta$ scans), and in-plane strain of the PMN-PT substrate (using a strain gauge), respectively. Prior to the measurements, a large dc electric field of $E=+10 \mathrm{kV} / \mathrm{cm}$ across the NSMO/PMNPT structure is applied for $30 \mathrm{~min}$ to ensure that the PMN-PT substrate is fully positively polarized.

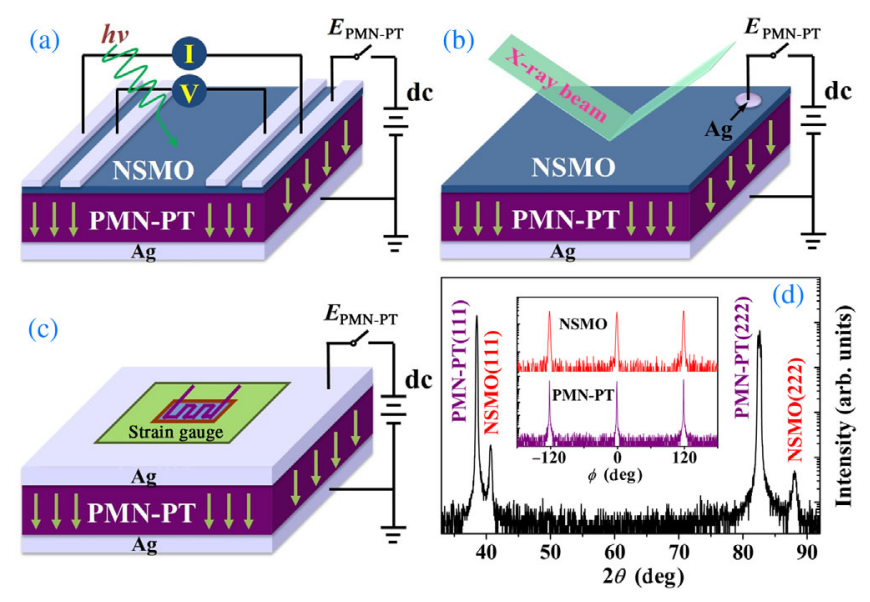

FIG. 1. Schematic of the experimental setups for in situ measurements of (a) film resistance, (b) out-of-plane strain, and (c) in-plane strain of the PMN-PT substrate, respectively. (d) XRD $\theta-2 \theta$ scan of the NSMO/PMN-PT structure. (Inset) XRD $\phi$ scans taken on the $\operatorname{NSMO(101)~and~PMN-PT(101)~}$ diffraction peaks.

\section{RESULTS AND DISCUSSION}

Figure 1(d) shows the XRD $\theta-2 \theta$ scan pattern for the asgrown NSMO/PMN-PT structure. The NSMO film is highly (111) oriented, without other impurity phases. In order to witness the in-plane epitaxial relationship between the film and the substrate, we perform XRD $\phi$ scans which are taken on the (101) diffraction peaks of the NSMO film and the PMN-PT substrate, respectively. As presented in the inset of Fig. 1(d), two sets of trifold symmetry are observed from the NSMO film and the PMN-PT substrate, respectively, revealing a good "cube-on-cube" heteroepitaxy. The initial strain state of the NSMO film due to lattice mismatch can be determined by analyzing its main peak in the XRD scan curve. The calculated out-of-plane lattice spacing $d_{111}$ (about $2.216 \AA$ ) is smaller than that of the bulk value (approximately $2.226 \AA$ ) [18,19], which indicates that the NSMO film suffers an out-of-plane compressive strain $(-0.45 \%)$. Using the Poisson relation $\delta \varepsilon_{z z}=-2 \nu /(1-\nu) \delta \varepsilon_{x x}[20]$ and Poisson's ratio $\nu=$ 0.37 [21], the in-plane tensile strain of the NSMO film can be estimated to be $0.38 \%$. This result corresponds to the smaller lattice constants of the NSMO bulk $(a \sim b \sim c \sim 3.855 \AA)$ [18,19] compared to those of the PMN-PT substrate $(a \sim b \sim c \sim 4.02 \AA)$.

Figure 2(a) displays in situ electric-field tuning of the resistance of the NSMO film under both bipolar and unipolar voltage cycling at room temperature using the experimental setup in Fig. 1(a). Upon cycling a large bipolar electric field with an amplitude of $E=$ $10 \mathrm{kV} / \mathrm{cm}$, a butterflylike loop of $\Delta R / R$ to $E$ is found. Here, $\Delta R / R$ is defined as $\Delta R / R=[R(E)-$ $\left.R\left(P_{r}^{+}\right)\right] / R\left(P_{r}^{+}\right)$. This finding is consistent with the typical butterflylike curve of the in-plane strain $\left(\delta \varepsilon_{x x(\mathrm{PMN}-\mathrm{PT})}\right)$ 

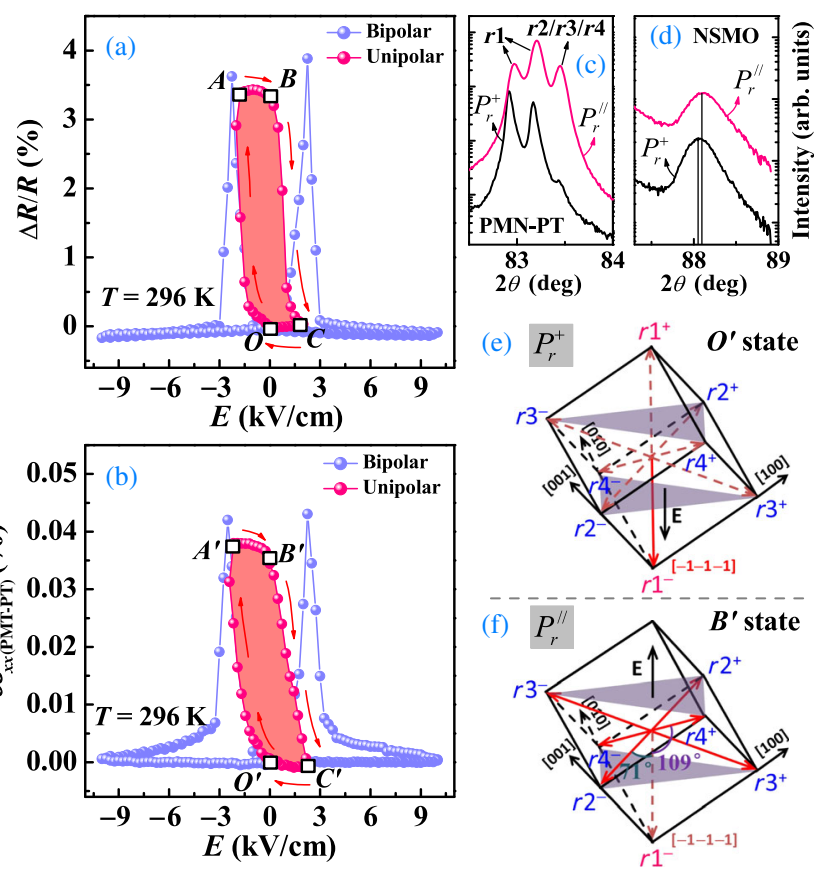

FIG. 2. Electric-field-induced (a) relative resistance change of the NSMO film and (b) the in-plane strain of the PMN-PT substrate as a function of bipolar and unipolar $E$ values applied across the PMN-PT substrate at $T=296 \mathrm{~K}$. (c),(d) XRD $\theta-2 \theta$ scans for the PMN-PT(222) substrate and the $\operatorname{NSMO(222)~film~}$ under the $P_{r}^{+}$and $P_{r}^{/ /}$states, respectively. (e),(f) Schematic diagrams of the polarization vectors in the rhombohedral phase for the $P_{r}^{+}$and $P_{r}^{/ /}$states of the PMN-PT, respectively.

versus the electric field for the PMN-PT substrate in Fig. 2(b), as measured using a strain gauge [see Fig. 1(c)], which clearly proves that the resistance evolution in the NSMO film is induced by the electric-field-induced lattice strain in the PMN-PT substrate. It is worth noting that once a small negative field $E=-2 \mathrm{kV} / \mathrm{cm}\left(|E|<E_{C(\mathrm{PMN}-\mathrm{PT})}\right)$ is applied to the positively poled PMN-PT substrate (i.e., the $\boldsymbol{O}^{\prime}$ or $P_{r}^{+}$state) and then removed, the film resistance is enhanced remarkably. Afterwards, by applying another small positive $E=+2 \mathrm{kV} / \mathrm{cm}$, the resistance can recover to the initial $\boldsymbol{O}$ state. This resistance change process can be attributed to the stable and reversible ferroelastic domain switching in the PMN-PT(111) substrate. In the rhombohedral PMN-PT crystal, the polarization vectors align along eight body-diagonal directions of the pseudocubic unit cell with four structural domains $(r 1, r 2, r 3, r 4)$. Upon applying a large positive $E=+10 \mathrm{kV} / \mathrm{cm}$, the PMN-PT substrate is fully poled, with the polarization pointing to $r 1^{-}$(i.e., the [ [ $\left.\begin{array}{lll}\overline{1} & \overline{1} & 1\end{array}\right]$ direction and the $P_{r}^{+}$state) [see Fig. 2(e)]. Then, after we apply a small negative $E=-2 \mathrm{kV} / \mathrm{cm}$, the PMN-PT substrate is partially depolarized. The polarization switching pathways include $109^{\circ}$ ferroelastic switching from $r 1^{-}$to $r 2^{+} / r 4^{+} / r 3^{-}$and $71^{\circ}$ ferroelastic switching from $r 1^{-}$to $r 2^{-} / r 4^{-} / r 3^{+}$. The $r 2 / r 3 / r 4$ domain structures are denoted by the $P_{r}^{/ /}$state in Fig. 2(f). This domain switching is accompanied by an in-plane tensile strain in the PMN-PT substrate and the NSMO film, as reflected by the diffraction peak shift to higher Bragg angles in Figs. 2(c) and 2(d) using in situ XRD measurements shown in Fig. 1(b). In particular, when the polarization direction is switched from the $P_{r}^{+}$to the $P_{r}^{/ /}$state, the PMN-PT(222) reflection splits from two peaks to three peaks. The peak corresponding to the $r 2 / r 3 / r 4$ domain structures reappears and the relative intensity of the peaks corresponding to the $r 1$ domain structure decreases dramatically, which further confirms the abovementioned $109^{\circ}$ and $71^{\circ}$ ferroelastic switching pathways. Accordingly, these two stable and switchable resistance states (the $\boldsymbol{O}$ and $\boldsymbol{B}$ states) can be achieved by reversing the applied voltage near the coercive field to dynamically rotate the polarization between the out-of-plane and in-plane directions in the PMN-PT substrates.

To obtain more remnant resistance states, we precisely adjust the negative amplitude of sweeping electric fields and present the results in Fig. 3(a). The presence of an intermediate resistance state between the $\boldsymbol{O}$ and $\boldsymbol{B}$ states could be linked to the different remnant in-plane strain states of the PMN-PT substrate [14,15,22,23]. As reported by our previous work [24], as the negative amplitude is reduced from $|-2.0 \mathrm{kV} / \mathrm{cm}|$ to $|-1.0 \mathrm{kV} / \mathrm{cm}|$, the relative intensity of the peak corresponding to the $r 2 / r 3 / r 4$ domain structures decreases gradually, which hints at the weakening of the $109^{\circ}$ and $71^{\circ}$ ferroelastic domain switching and the resultant reduction in remnant in-plane tensile strain of the PMN-PT substrate. On the basis of
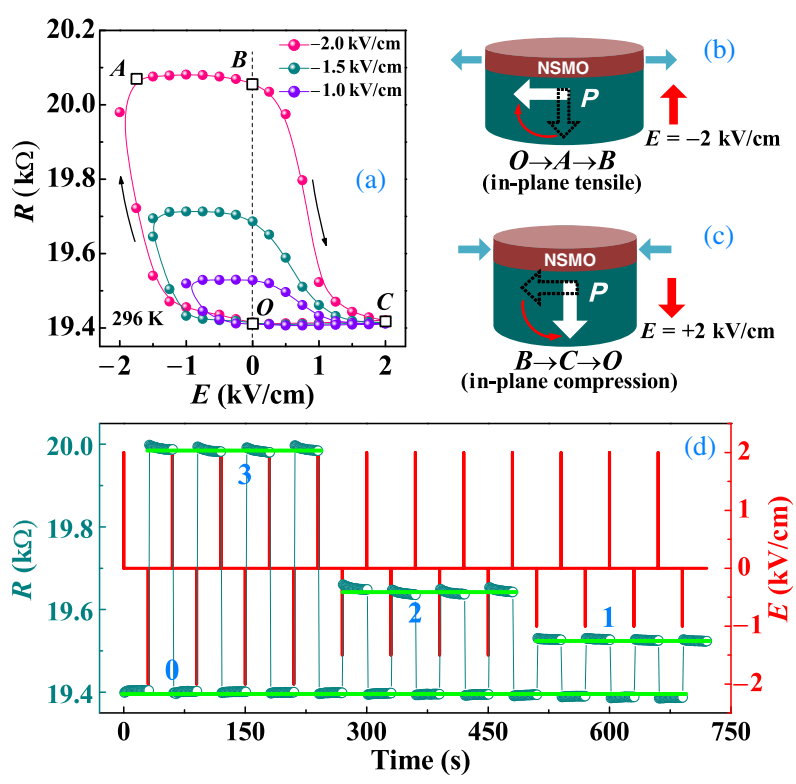

FIG. 3. (a) Resistance hysteresis loops when cycling the electric field between positive maximum and several negative values at $T=296 \mathrm{~K}$. (b),(c) Schematic diagrams for the ferroelastic domain switching. (d) Nonvolatile resistance switching of the NSMO film by a sequence of pulse electric fields. 
Fig. 3(a), we demonstrate a rudimental manifestation of the applicability of such an electrically driven ferroelastic strain effect for an energy-efficient information storage element. As depicted in Fig. 3(d), the nonvolatile logic states are written by applying electric-field pulses with an amplitude of $E=+2.0 \mathrm{kV} / \mathrm{cm}$ for the 0 state and $E=-1.0,-1.5$, and $-2.0 \mathrm{kV} / \mathrm{cm}$ for the 1,2 , and 3 states, respectively. It should be pointed out that a reset step by applying an impulse field of $E=+2 \mathrm{kV} / \mathrm{cm}$ is required to set the polarization vectors to the downward direction with the minimum resistance state before writing to different higher-resistance values. Under such circumstances, the resistance states stored in this device are reversible and nonvolatile, and they can be read out in a nondestructive manner. The domain-engineered ferroelastic switching [see Figs. 3(b) and 3(c)] provides a framework for designing the next-generation low-energy-consumption, high-density nonvolatile memory devices.

Figure 4 shows the temperature dependence of the resistance for the NSMO film in the dark and under light illumination when the PMN-PT substrate is in the $P_{r}^{+}$and $P_{r}^{/ /}$states, respectively. For the $P_{r}^{+}$state in the dark, the resistance increases with decreasing temperature and undergoes a charge-ordering phase transition at $T_{\mathrm{CO}} \sim 127 \mathrm{~K}$. Here, $T_{\mathrm{CO}}$ can be derived from the $d(\ln R) / d\left(T^{-1}\right)-T$ curve [25]. Associated with the polarization switching from the $P_{r}^{+}$to the $P_{r}^{/ /}$state, a visible increase in the resistance and $T_{\mathrm{CO}}$ [see Fig. 4(a)] is observed, which is due to the in-plane tensile straininduced enhancement of the electron-lattice coupling strength and suppression of the double-exchange

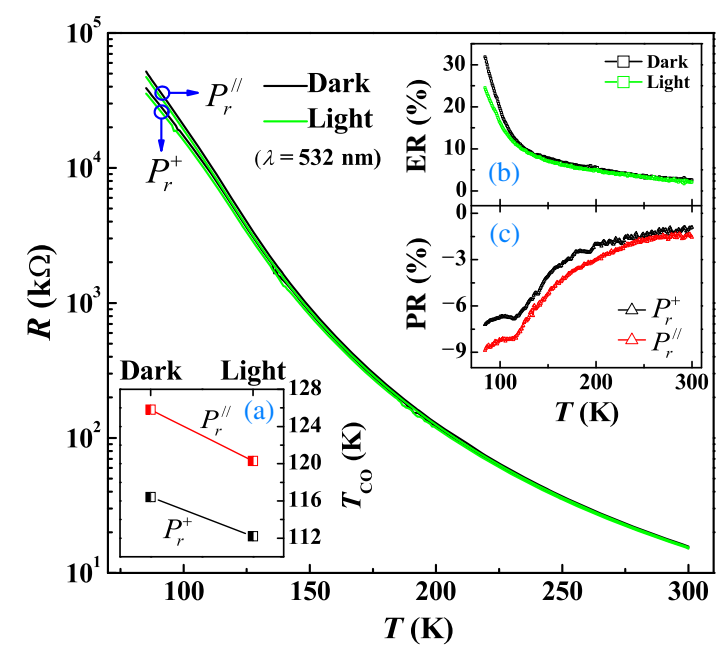

FIG. 4. Temperature dependence of the resistance for the NSMO film in the dark and under light illumination when the PMN-PT substrate is in the $P_{r}^{+}$and $P_{r}^{/ /}$states, respectively. (a) The corresponding $T_{\mathrm{CO}}$ values. (b),(c) Temperaturedependent electroresistance (ER) in the dark and under light illumination, and photoresistance (PR) under the $P_{r}^{+}$and $P_{r}^{/ /}$ states, respectively. interaction [26]. This result implies that the in-plane tensile strain favors the $\mathrm{CO}$ insulating phase but destabilizes the FM metallic phase. Moreover, whether for the $P_{r}^{+}$or $P_{r}^{/ /}$ state, both the film resistance and $T_{\mathrm{CO}}$ are reduced upon light illumination. Light can generate more carries and enhanced hopping of small polarons, leading to the delocalization of $e_{g}$ carries and reduction of the resistance $[16,17,27]$. On the other hand, light can also excite spin-down $e_{g}$ electrons in the FM phase and destroy the FM coupling between spin-up $e_{g}$ and $t_{2 g}$ electrons in $\mathrm{Mn}^{3+}$ ions, thereby increasing the resistance [28,29]. In our case, the fraction of the FM phase is small due to the in-plane tensile strain, and thus the photoinduced delocalization effect in the $\mathrm{CO}$ phase rather than the demagnetization effect in the FM phase plays a primary role in determining the resistance. To unveil the coupling effect of the light and the electric field in the NSMO/PMN-PT structure, we plot temperature-dependent electroresistance (ER) in the dark and under light illumination in Fig. 4(b). Here, ER is defined as ER $=\left[R\left(P_{r}^{/ /}\right)-R\left(P_{r}^{+}\right)\right] / R\left(P_{r}^{+}\right)$. In the dark, ER increases upon cooling and reaches a maximal value of $31.4 \%$ at $T=85 \mathrm{~K}$, suggesting the high sensitivity of the $\mathrm{CO}$ phase to the lattice strain at low temperature. We note that the ER of the film is greatly reduced over the whole temperature range after light illumination. For example, the ER at $T=85 \mathrm{~K}$ is lowered from $31.4 \%$ in the dark to $23.9 \%$ under light illumination. Such an optically tunable electroresistance response can be ascribed to the photoexcited delocalization of $e_{g}$ carries and the resulting reduction of the $\mathrm{CO}$ phase, which suppresses the ferroelastic strain effect of the NSMO/PMN-PT structure. The mutual interaction between the electric-field and lightinduced effects is manifested not only by the lightcontrolled electroresistance effect but also by the electric-field-controlled photoresistance (PR) effect. The latter is shown in Fig. 4(c), where the PR of the NSMO film is measured under the $P_{r}^{+}$and $P_{r}^{/ /}$states. Here, PR is defined as PR $=\left(R_{\text {light }}-R_{\text {dark }}\right) / R_{\text {dark }}$. For the $P_{r}^{+}$state, the negative PR increases in magnitude as $T$ decreases and the PR- $T$ curve shows an appreciable kink near $T_{\mathrm{CO}}$. When the polarization is rotated from the $P_{r}^{+}$to the $P_{r}^{/ /}$state, the magnitude of the PR is enhanced considerably, especially at low temperatures. For example, the relative change in PR, $\Delta \mathrm{PR} / \mathrm{PR}=\left[\mathrm{PR}\left(P_{r}^{/ /}\right)-\mathrm{PR}\left(P_{r}^{+}\right)\right] / \mathrm{PR}\left(P_{r}^{+}\right)$, reaches $22.6 \%$ at $T=85 \mathrm{~K}$. The electrically tunable photoresistance effect is closely related to the coexisting $\mathrm{CO}$ insulating and FM metallic phases, i.e., the phase separation. At the lowtemperature $\mathrm{CO}$ state, e.g., $T=85 \mathrm{~K}$, the $\mathrm{CO}$ phase coexists with the FM phase, with the former dominating over the latter, where the PR arises from the light-induced conversion of the $\mathrm{CO}$ phase to the FM phase. The giant enhancement in the resistance (e.g., ER $\sim 31.4 \%$ at $T=85 \mathrm{~K}$ ) induced by ferroelastic (in-plane tensile) strain [see Fig. 4(b)] means that a significant fraction of the FM 
metallic phase has been converted into the $\mathrm{CO}$ insulating phase due to the expansion of the film in-plane lattice. Namely, the fraction of the CO insulating phase in the $P_{r}^{/ /}$ state is larger than that in the $P_{r}^{+}$state. Consequently, the effective fraction of the $\mathrm{CO}$ insulating phase that is converted to the FM metallic phase in the $P_{r}^{/ /}$state would be larger than that in the $P_{r}^{+}$state. Thus, the PR in the $P_{r}^{/ /}$ state is larger than that in the $P_{r}^{+}$state. All of these data directly disclose that the electric-field effect strongly couples with the light effect, which is deeply mediated by electronic phase separation.

To further substantiate the role played by phase separation in the correlation between the electric-field and lightinduced effects, we monitored the resistance switching of the NSMO film in the dark and under light illumination by switching the polarization between the $P_{r}^{+}$and $P_{r}^{/ /}$states at room temperature, as shown in Fig. 5(a). Upon applying a sequence of $E= \pm 2 \mathrm{kV} / \mathrm{cm}$ pulse electric field to the PMN-PT, the film resistance responds sharply and can be reversibly switched. The effect of the light on the electroresistance is evident in Fig. 5(b), where the ER is plotted against time in the dark and under light illumination. Obviously, the ER is suppressed under light illumination, which is commensurate with the temperature-dependent results [see Fig. 4(b)] and stems from the light-induced reduction of the PM insulating phase at room temperature. Again, to identify the response of the electric field to the
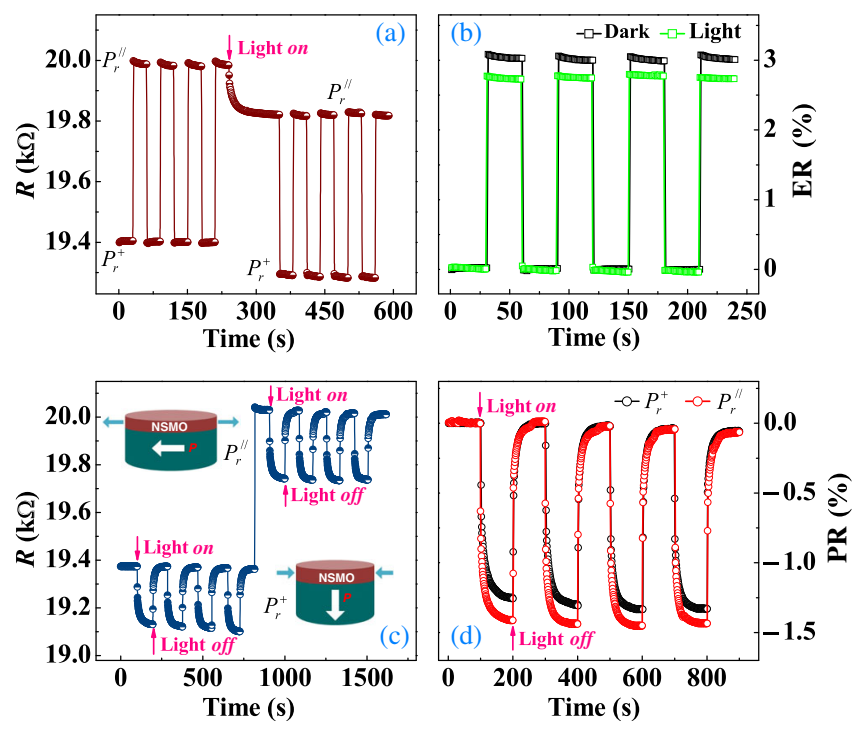

FIG. 5. (a) Resistance switching of the NSMO film in the dark and under light illumination by switching the polarization between the $P_{r}^{+}$and $P_{r}^{/ /}$states at $T=296 \mathrm{~K}$. (b) ER as a function of time in the dark and under light illumination. (c) Resistance switching of the NSMO film by turning the light on and off under the $P_{r}^{+}$and $P_{r}^{/ /}$states. (Insets) Schematic diagrams for the ferroelastic strain effect. (d) PR as a function of time under the $P_{r}^{+}$and $P_{r}^{/ /}$states. photoresistance, we record the resistance of the NSMO film by turning the light on and off under the $P_{r}^{+}$and $P_{r}^{/ /}$states [see the insets of Fig. 5(c)] at room temperature in Fig. 5(c). We find that the resistance exhibits the photoinduced relaxation character with the light on and then restores the original value after the light is off, which is caused by the delocalization relaxation process and thermal fluctuation, respectively [27]. According to Fig. 5(c), the PR of the NSMO film is calculated for the $P_{r}^{+}$and $P_{r}^{/ /}$states, respectively. As depicted in Fig. 5(d), it is apparent that the electric-field-induced ferroelastic (in-plane tensile) strain increases the magnitude of the PR, signaling electrically induced conversion of the FM phase into the PM phase. These findings together explicitly establish that the electricfield and light-induced effects strongly correlate with each other and are essentially driven by the fraction of the PM phase relative to the FM phase. Based on Figs. 4 and 5, it is concluded that, whether the film is under the $\mathrm{CO}$ or the PM state, the electronic phase separation is a crucial ingredient that accounts for the coupling effect of the light and the electric field. Additionally, the heat effect always is a challenging problem and is difficult to separate from the optical-electric effect under light illumination. In our experiments, two factors can be considered to ignore the heat effect. First, since the photon energy $(2.33 \mathrm{eV})$ of 532 $\mathrm{nm}$ light used in the experiment is larger than the band gap of manganites $(1.0-1.3 \mathrm{eV})$, the light can be absorbed by the NSMO films, and thus the optical-electric effect is dominant. Second, the sample is mounted on a $\mathrm{Cu}$ cryostat through the silver paint. If light illumination creates a little heat, the heat can also be dissipated into the holder, and the sample remains at a fixed temperature due to the closedcycle cryostat. Therefore, the heating effect can be precluded.

\section{CONCLUSIONS}

In summary, we report in this paper on the electric-field and light-dependent cocontrol of phase-separation-driven functionalities using a simple NSMO/PMN-PT multiferroic heterostructure. The distinct stable and nonvolatile resistance states can be reversibly switched by applying appropriate voltage pulses, which is closely related to the electric-field-induced $109^{\circ}$ and $71^{\circ}$ ferroelastic domain switching and induces dissimilar in-plane strain states. Such an electroresistance response is found to be optically tunable. Moreover, the photoexcited delocalization of $e_{g}$ carriers gives rise to a noticeable reduction in the film resistance. The photoresistance effect can be enhanced by $22.6 \%$ through the electric-field-induced ferroelastic strain. This result, together with the optically controlled electroresistance response, reveals intimate coupling between the electric-field and light-induced effects, which can be plausibly interpreted in terms of the phase separation model. Our findings provide a unique approach to realize 
multifield tuning of electronic phase separation in complex oxide heterostructures, which allows for the integration of extra functionality (e.g., a light sensor) in nonvolatile memory systems.

\section{ACKNOWLEDGMENTS}

This work was supported by the National Key Project for Basic Research (Grant No. 2014CB921002), the National Natural Science Foundation of China (Grants No. 11374225, No. 11574227, No. 51572280, and No. 11504432), the Foundation of the Shanghai Committee for Science and Technology (Grant No. 15JC1403600), the Fundamental Research Funds for the Central Universities (Grant No. 18CX02046A), the Research Grant Council of Hong Kong (Project Nos. HKU 702112P and HKU 701813), and the Qingdao Science and Technology Program for Youth (Grant No. 16-5-1-6-jch).

[1] P. A. Lee, N. Nagaosa, and X.-G. Wen, Doping a Mott insulator: Physics of high-temperature superconductivity, Rev. Mod. Phys. 78, 17 (2006).

[2] M. Uehara, S. Mori, C. H. Chen, and S.-W. Cheong, Percolative phase separation underlies colossal magnetoresistance in mixed-valent manganites, Nature (London) 399, 560 (1999).

[3] V. Garcia and M. Bibes, Ferroelectric tunnel junctions for information storage and processing, Nat. Commun. 5, 4289 (2014).

[4] N. Takubo, I. Onishi, K. Takubo, T. Mizokawa, and K. Miyano, Photoinduced Metal-to-Insulator Transition in a Manganite Thin Film, Phys. Rev. Lett. 101, 177403 (2008).

[5] L. W. Zhang, C. Israel, A. Biswas, R. L. Greene, and A. de Lozanne, Direct observation of percolation in a manganite thin film, Science 298, 805 (2002).

[6] T. Z. Ward, J. D. Budai, Z. Gai, J. Z. Tischler, L. F. Yin, and J. Shen, Elastically driven anisotropic percolation in electronic phase-separated manganites, Nat. Phys. 5, 885 (2009).

[7] M. H. Burkhardt, M. A. Hossain, S. Sarkar, Y.-D. Chuang, A. G. C. Gonzalez, A. Doran, A. Scholl, A. T. Young, N. Tahir, Y. J. Choi, S.-W. Cheong, H. A. Dürr, and J. Stöhr, Imaging the First-Order Magnetic Transition in $\mathrm{La}_{0.35} \mathrm{Pr}_{0.275} \mathrm{Ca}_{0.375} \mathrm{MnO}_{3}$, Phys. Rev. Lett. 108, 237202 (2012).

[8] T. Elovaara, S. Majumdar, H. Huhtinen, and P. Paturi, Photoinduced colossal magnetoresistance under substantially reduced magnetic field, Adv. Funct. Mater. 25, 5030 (2015).

[9] M. Zheng, M. M. Yang, Q. X. Zhu, X. Y. Li, G. Y. Gao, R. K. Zheng, Y. Wang, X. M. Li, X. Shi, H. S. Luo, and X.G. Li, Tunable interface strain coupling and its impact on the electronic transport and magnetic properties of $\mathrm{La}_{0.5} \mathrm{Ca}_{0.5} \mathrm{MnO}_{3} / \mathrm{Pb}\left(\mathrm{In}_{1 / 2} \mathrm{Nb}_{1 / 2}\right) \mathrm{O}_{3}-\mathrm{Pb}\left(\mathrm{Mg}_{1 / 3} \mathrm{Nb}_{2 / 3}\right) \mathrm{O}_{3}-$ $\mathrm{PbTiO}_{3}$ multiferroic heterostructures, Phys. Rev. B 90, 224420 (2014).
[10] M. Zheng and R. K. Zheng, Electric-Field-Tunable Ferroelastic Control of Nonvolatile Resistivity and Ferromagnetic Switching in Multiferroic $\mathrm{La}_{0.67} \mathrm{Ca}_{0.33} \mathrm{MnO}_{3} /$ $\left[\mathrm{PbMg}_{1 / 3} \mathrm{Nb}_{2 / 3} \mathrm{O}_{3}\right]_{0.7}\left[\mathrm{PbTiO}_{3}\right]_{0.3}$ Heterostructures, Phys. Rev. Applied 5, 044002 (2016).

[11] C. Thiele, K. Dörr, O. Bilani, J. Rödel, and L. Schultz, Influence of strain on the magnetization and magnetoelectric effect in $\mathrm{La}_{0.7} A_{0.3} \mathrm{MnO}_{3} / \mathrm{PMN}-\mathrm{PT}(001)(A=\mathrm{Sr}, \mathrm{Ca})$, Phys. Rev. B 75, 054408 (2007).

[12] R. K. Zheng, Y. Jiang, Y. Wang, H. L. W. Chan, C. L. Choy, and H.S. Luo, Ferroelectric poling and converse-piezoelectric-effect-induced strain effects in $\mathrm{La}_{0.7} \mathrm{Ba}_{0.3} \mathrm{MnO}_{3}$ thin films grown on ferroelectric singlecrystal substrates, Phys. Rev. B 79, 174420 (2009).

[13] Q. P. Chen, J. J. Yang, Y. G. Zhao, S. Zhang, J. W. Wang, M. H. Zhu, Y. Yu, X. Z. Zhang, Z. Wang, B. Yang, D. Xie, and T. L. Ren, Electric-field control of phase separation and memory effect in $\mathrm{Pr}_{0.6} \mathrm{Ca}_{0.4} \mathrm{MnO}_{3} / \mathrm{Pb}\left(\mathrm{Mg}_{1 / 3} \mathrm{Nb}_{2 / 3}\right)_{0.7} \times$ $\mathrm{Ti}_{0.3} \mathrm{O}_{3}$ heterostructures, Appl. Phys. Lett. 98, 172507 (2011).

[14] Y. J. Yang, Z. L. Luo, M. M. Yang, H. L. Huang, H. B. Wang, J. Bao, G. Q. Pan, C. Gao, Q. Hao, S. T. Wang, M. Jokubaitis, W. Z. Zhang, G. Xiao, Y. P. Yao, Y. K. Liu, and X. G. Li, Piezo-strain induced non-volatile resistance states in (011) $-\mathrm{La}_{2 / 3} \mathrm{Sr}_{1 / 3} \mathrm{MnO}_{3} / 0.7 \mathrm{~Pb}\left(\mathrm{Mg}_{2 / 3} \mathrm{Nb}_{1 / 3}\right) \mathrm{O}_{3}-0.3 \mathrm{PbTiO}_{3}$ epitaxial heterostructures, Appl. Phys. Lett. 102, 033501 (2013).

[15] W. P. Zhou, Y. Q. Xiong, Z. M. Zhang, D. H. Wang, W. S. Tan, Q. Q. Cao, Z. H. Qian, and Y. W. Du, Multilevel resistance switching memory in $\mathrm{La}_{2 / 3} \mathrm{Ba}_{1 / 3} \mathrm{MnO}_{3} /$ $0.7 \mathrm{~Pb}\left(\mathrm{Mg}_{1 / 3} \mathrm{Nb}_{2 / 3}\right) \mathrm{O}_{3}-0.3 \mathrm{PbTiO}_{3} \quad$ (011) heterostructure by combined straintronics-spintronics, ACS Appl. Mater. Interfaces 8, 5424 (2016).

[16] E. J. Guo, J. Gao, and H. B. Lu, Strain-mediated electricfield control of photoinduced demagnetization in $\mathrm{La}_{0.8} \mathrm{Ca}_{0.2} \mathrm{MnO}_{3}$ thin films, Appl. Phys. Lett. 98, 081903 (2011).

[17] W. Li, X. L. Dong, S. H. Wang, and K. X. Jin, Electricfield modulation of photoinduced effect in phase-separated $\operatorname{Pr}_{0.65}\left(\mathrm{Ca}_{0.75} \mathrm{Sr}_{0.25}\right)_{0.35} \mathrm{MnO}_{3} / \mathrm{PMN}-\mathrm{PT}$ heterostructure, Appl. Phys. Lett. 109, 091907 (2016).

[18] F. Millange, V. Caignaert, G. Mather, E. Suard, and B. Raveau, Low temperature orthorhombic to monoclinic transition due to size effect in $\mathrm{Nd}_{0.7} \mathrm{Ca}_{0.3-x} \mathrm{Sr}_{x} \mathrm{MnO}_{3}$ : Evidence for a new type of charge ordering, J. Solid State Chem. 127, 131 (1996).

[19] S. W. Jin, G. Y. Gao, Z. Z. Yin, Z. Huang, X. Y. Zhou, and W. B. Wu, Strain state evolution and thickness-dependent properties of epitaxial $\mathrm{Nd}_{0.7} \mathrm{Sr}_{0.3} \mathrm{MnO}_{3}$ films, Phys. Rev. B 75, 212401 (2007).

[20] S. P. Timoshenko and J. N. Goodier, Theory of Elasticity (McGraw-Hill, New York, 1987).

[21] C. Adamo, X. Ke, H. Q. Wang, H. L. Xin, T. Heeg, M. E. Hawley, W. Zander, J. Schubert, P. Schiffer, D. A. Muller, L. Maritato, and D. G. Schlom, Effect of biaxial strain on the electrical and magnetic properties of (001) $\mathrm{La}_{0.7} \mathrm{Sr}_{0.3} \mathrm{MnO}_{3}$ thin films, Appl. Phys. Lett. 95, 112504 (2009).

[22] M. Liu, B. M. Howe, L. Grazulis, K. Mahalingam, T. X. Nan, N. X. Sun, and G. J. Brown, Voltage-impulse-induced non-volatile ferroelastic switching of ferromagnetic 
resonance for reconfigurable magnetoelectric microwave devices, Adv. Mater. 25, 4886 (2013).

[23] B. W. Zhi, G. Y. Gao, H. R. Xu, F. Chen, X. L. Tan, P. F. Chen, L. F. Wang, and W. B. Wu, Electric-field-modulated nonvolatile resistance switching in $\mathrm{VO}_{2} / \mathrm{PMN}-\mathrm{PT}(111)$ heterostructures, ACS Appl. Mater. Interfaces 6, 4603 (2014).

[24] M. Zheng, H. Ni, Y. P. Qi, W. Y. Huang, J. L. Zeng, and J. Gao, Ferroelastic strain control of multiple nonvolatile resistance tuning in $\mathrm{SrRuO}_{3} / \mathrm{PMN}-\mathrm{PT}(111)$ multiferroic heterostructures, Appl. Phys. Lett. 110, 182403 (2017).

[25] A. P. Ramirez, P. Schiffer, S.-W. Cheong, C. H. Chen, W. Bao, T. T. M. Palstra, P. L. Gammel, D. J. Bishop, and B. Zegarski, Thermodynamic and Electron Diffraction Signatures of Charge and Spin Ordering in $\mathrm{La}_{1-x} \mathrm{Ca}_{x} \mathrm{MnO}_{3}$, Phys. Rev. Lett. 76, 3188 (1996).

[26] Q. X. Zhu, M. Zheng, M. M. Yang, X. M. Li, Y. Wang, X. Shi, H. L. W. Chan, H. S. Luo, X. G. Li, and R. K. Zheng,
Effects of ferroelectric-poling-induced strain on magnetic and transport properties of $\mathrm{La}_{0.67} \mathrm{Ba}_{0.33} \mathrm{MnO}_{3}$ thin films grown on (111)-oriented ferroelectric substrates, Appl. Phys. Lett. 103, 132910 (2013).

[27] S. G. Zhao, K. X. Jin, and C. L. Chen, Photoinduced effect in charge-ordering $\mathrm{La}_{0.5} \mathrm{Ca}_{0.5} \mathrm{MnO}_{3}$ film, J. Appl. Phys. 101, 083701 (2007).

[28] Y. G. Zhao, J. J. Li, R. Shreekala, H. D. Drew, C. L. Chen, W. L. Cao, C. H. Lee, M. Rajeswari, S. B. Ogale, R. Ramesh, G. Baskaran, and T. Venkatesan, Ultrafast Laser Induced Conductive and Resistive Transients in $\mathrm{La}_{0.7} \mathrm{Ca}_{0.3} \mathrm{MnO}_{3}$ : Charge Transfer and Relaxation Dynamics, Phys. Rev. Lett. 81, 1310 (1998).

[29] K. Matsuda, A. Machida, Y. Moritomo, and A. Nakamura, Photoinduced demagnetization and its dynamical behavior in a $\left(\mathrm{Nd}_{0.5} \mathrm{Sm}_{0.5}\right)_{0.6} \mathrm{Sr}_{0.4} \mathrm{MnO}_{3}$ thin film, Phys. Rev. B 58, R4203 (1998). 